\title{
Equilibrium and equilibration in a gluon plasma with improved matrix elements
}

\author{
Bin Zhang ${ }^{1, a}$ \\ ${ }^{1}$ Department of Chemistry and Physics, Arkansas State University, P.O. Box 419, State University, AR \\ 72467-0419, U.S.A.
}

\begin{abstract}
The hot and dense matter created in the early stage of a relativistic heavy ion collision is composed mainly of gluons. Radiative processes can play an important role for the thermalization of such partonic systems. The simplest parton number changing processes are commonly described by the Gunion-Bertsch formula. We show that the cross section from the exact matrix element for the lowest order radiative process could be significantly smaller than that based on the Gunion-Bertsch formula. In light of this, we discuss the role of radiative processes on the equilibrium and equilibration of a gluon plasma.
\end{abstract}

\section{Introduction}

Relativistic heavy ion collisions have been used to produce and study matter under extreme conditions similar to those existed in the early Universe. Many spectacular properties of hot and dense nuclear matter have been observed. With the advancement of the hydrodynamical and related simulations, people were able to recognize that the quark and gluon system quickly thermalizes. The resultant Quark-Gluon Plasma, along with some cold atomic systems, evolves with the lowest ever observed shear viscosity to entropy density ratio [1]. Intensive research efforts have been directed toward understanding these observations [2-10]. Some recent microscopic studies focused on contributions from particle number changing processes [11-17]. The Gunion-Bertsch formula [18, 19] is widely used to study the lowest order radiative process. In the following, we will discuss our recent attempts at performing simulations beyond the Gunion-Bertsch formula.

\section{Results and discussions}

Our focus will be on the gluons as they form the dominant component in the initial stage and they interact more strongly compared with the quarks. Instead of using the Gunion-Bertsch formula, our calculations will start from the exact formula $[20,21]$ for the lowest order radiative process. In this case, the matrix element modulus squared can be determined from the inner-products of particle fourmomenta by

$$
\left|M_{g g \rightarrow g g g}\right|^{2}=\frac{g_{s}^{6} N_{c}^{3}}{2\left(N_{c}^{2}-1\right)} \frac{\sum(i j)^{4} \sum(i j k l m)}{\prod(i j)}
$$

\footnotetext{
a e-mail: bzhang@astate.edu
} 
Here, $g_{s}$ is the strong interaction coupling constant, and $N_{c}$ is the number of colors. The string $(i j k l m)=(i j)(j k)(k l)(l m)(m i)$ where $(i j)$ is the inner-product of the four-momenta of particles $i$ and $j$. The sums and product are over all distinct permutations of particle labels, and the average over initial and sum over final internal (spin and color) degrees of freedom have been performed. The singularities in the denominator come from propagators. We will regulate these propagators by the Debye screening mass squared. Calculations can be done for a typical gluon plasma like that produced in the early stage of a heavy ion collision. When the strong interaction fine structure constant $\alpha_{s}=g_{s}^{2} /(4 \pi)=0.47$, the Debye screening mass squared $\mu^{2}=10 \mathrm{fm}^{-2}$, the two-particle center-of-mass energy squared $s=4$ $\mathrm{GeV}^{2}$, the calculated two-to-two elastic scattering cross section $\sigma_{22}=9 \pi \alpha_{s}^{2} /\left(2 \mu^{2}\right)=0.312 \mathrm{fm}^{2}$, while the two-to-three cross section $\sigma_{23}=0.0523 \mathrm{fm}^{2}$. The ratio $\sigma_{23} / \sigma_{22}=16.8 \%$ and is significantly smaller than the ratios from the Gunion-Bertsch formula based calculations which are about $50 \%$ [2]. When $\alpha_{s}$ is changed to 0.3 , the corresponding $\mu^{2}=6.38 \mathrm{fm}^{-2}$. This leads to $\sigma_{22}=0.199 \mathrm{fm}^{2}$ and $\sigma_{23}=0.0504 \mathrm{fm}^{2}$. Again, the ratio is much smaller than 50\%. The Dalitz plot of the outgoing particles shows that they are close to isotropically distributed. However, the reaction integral and the outgoing particle distribution can be very different from isotropic for the inverse process.

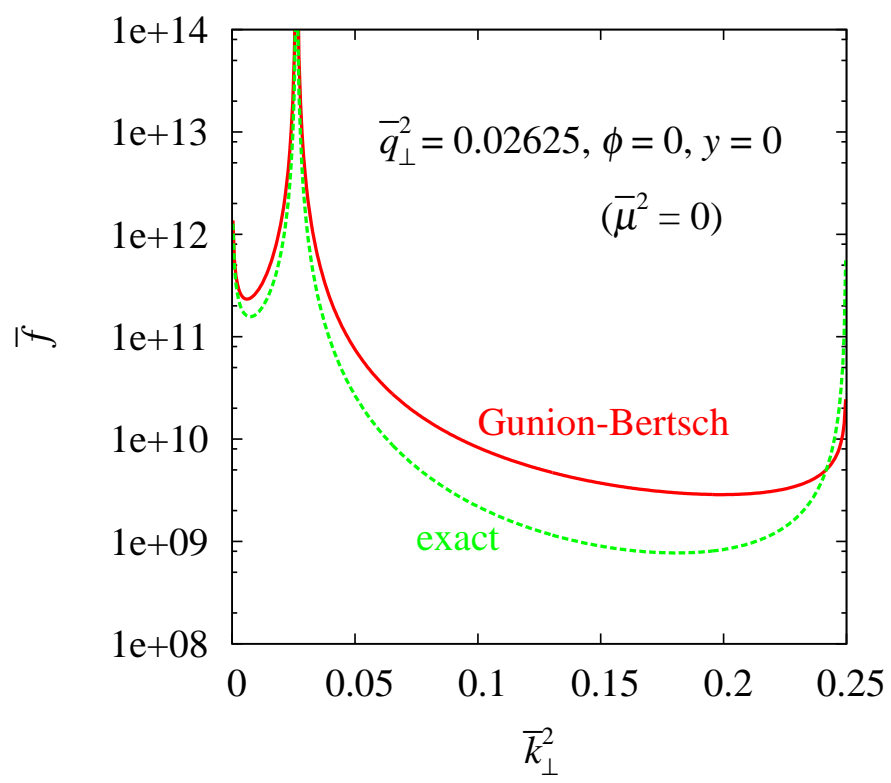

Figure 1. Comparison of results from the Gunion-Bertsch formula and the exact formula. The quantities are defined in the text.

It is interesting to see how the exact formula based calculations compare with the Gunion-Bertsch formula based ones in different phase space regions. The Gunion-Bertsch formula can be expressed in terms of the transverse momentum transfer $\vec{q}_{\perp}$ and the transverse momentum of the radiated gluon $\vec{k}_{\perp}$ as

$$
\left|M_{g g \rightarrow g g g}^{G B}\right|^{2}=\frac{9 g_{s}^{4} s^{2}}{2\left(q_{\perp}^{2}\right)^{2}} \frac{12 g_{s}^{2} q_{\perp}^{2}}{k_{\perp}^{2}\left(\vec{k}_{\perp}-\vec{q}_{\perp}\right)^{2}} .
$$

The singularities in the above formula can also be regulated by the Debye screening mass squared. The comparison can be done for $f\left(q_{\perp}, k_{\perp}, y, \phi\right)=\sum_{y_{1 a}^{\prime}, y_{1 b}^{\prime}}|M|^{2} /\left|\partial F / \partial y_{1}^{\prime}\right|_{F=0}$. Here $y$ is the rapidity of the radiated gluon, and $\phi$ is the angle between $\vec{q}_{\perp}$ and $\vec{k}_{\perp} . \quad y_{1 a}^{\prime}$ and $y_{1 b}^{\prime}$ are the two $y_{1}^{\prime}$ values that solve $F=0$, where $y_{1}^{\prime}$ is the rapidity of momentum transfer $\vec{q}$, and $F=0$ is the mass shell condition for the third outgoing particle (other than the radiated one or the momentum transferred 
one). $f$ can be obtained by integrating out the energy-momentum conserving delta function when calculating the cross section or the rate per unit volume. Therefore, $f$ is proportional to the differential cross section, and it reflects the outgoing particle distribution. For any given set of $q_{\perp}, k_{\perp}, y, \phi$ values, there are two sets of outgoing particles that satisfy energy-momentum conservation. When $y=0$, they are related by the mirror symmetry with respect to the transverse plane, and the two configurations give the same exact matrix element modulus squared. When $y \neq 0$, they generally give different exact matrix elements. In this respect, $f$ allows a one-to-one comparison between the Gunion-Bertsch formula and the exact formula. Since $f$ is proportional to $\alpha_{s}^{3} / s^{2}$, it is helpful to use the dimensionless quantity $\bar{f}=f /\left(\alpha_{s}^{3} / s^{2}\right)$. Likewise, one can also rescale $q_{\perp}, k_{\perp}$, and $\mu$ by $\sqrt{s}$ to obtain their dimensionless counterparts $\bar{q}_{\perp}, \bar{k}_{\perp}$, and $\bar{\mu}$. Fig. 1 compares $\bar{f}$ from the Gunion-Bertsch formula and from the exact formula. The matrix elements were not regulated. It is clear that $\bar{f}$ from the exact formula is smaller than that from the Gunion-Bertsch formula anywhere but close to the kinematic boundary at $\bar{k}_{\perp}^{2}=0.25$. The ratio increases from 1 at $\bar{k}_{\perp}^{2}=0$ to about 4 at $\bar{k}_{\perp}^{2} \sim 0.15$ and then drops down to 0 as $\bar{k}_{\perp}^{2} \rightarrow 0.25$. This behavior cannot be accounted for by the recently proposed correction factor for the Gunion-Bertsch formula [16]. At central rapidity $(y=0)$, the ratio is not sensitive to $\bar{q}_{\perp}^{2}$, while the dependence on $\bar{q}_{\perp}^{2}$ can be large at other places. A one-to-one comparison of the matrix element modulus squared can be done at $y=0$. As expected, the Gunion-Bertsch formula decreases continuously as $\bar{k}_{\perp}^{2} \rightarrow 0.25$, missing the collinear singularity at $\bar{k}_{\perp}^{2}=0.25$. Comparisons in other kinematic regions and with regulators can also be done, and the Gunion-Bertsch formula and the exact formula do not always agree.

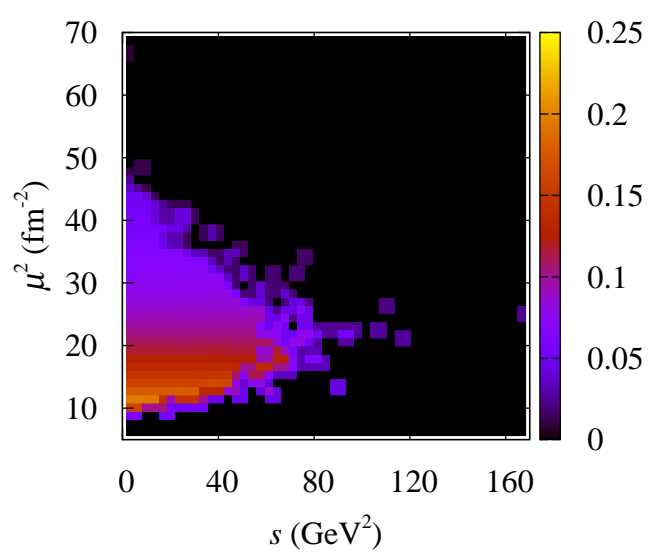

Figure 2. The elastic cross section as a function of the Debye mass squared and the center of mass energy squared. The colors of isolated points are from averages of adjacent points and do not reflect the values at those points.

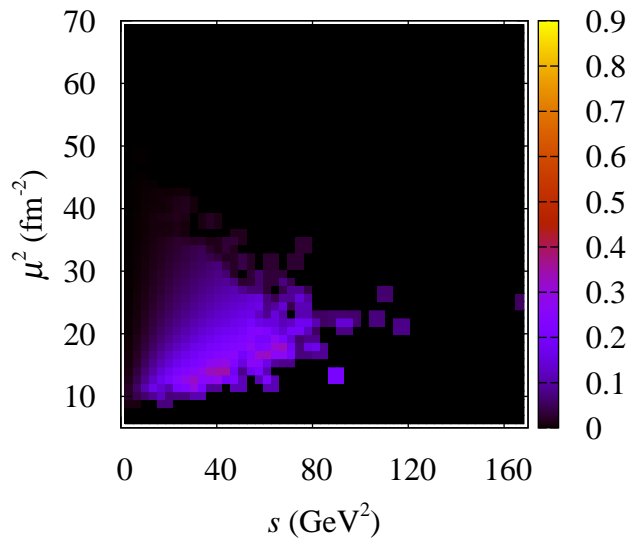

Figure 3. Same as Fig. 2 but for the 2 to 3 cross section.

In order to study the effect on the equilibration of a parton system, the exact matrix element was implemented into our radiative transport model. As a first step, we can look at the cross sections and rates in equilibrium. In the following, we will show some preliminary results and discussion their implications. The temperature will be set to $T=0.524 \mathrm{GeV}$, and the strong interaction fine structure constant $\alpha_{s}=0.4$. It turns out that the 2 to 3 cross section averaged over all 2 to 3 collisions is not that small relative to the 2 to 2 cross section averaged over all 2 to 2 collisions. The ratio, $\left\langle\sigma_{23}\right\rangle_{23} /\left\langle\sigma_{22}\right\rangle_{22} \sim 57 \%$. However, if both the radiative and the elastic cross sections are measured 
for every collision, the ratio $\left\langle\sigma_{23}\right\rangle /\left\langle\sigma_{22}\right\rangle \sim 17 \%$, significantly smaller than the biased ratio of $57 \%$. $\left\langle\sigma_{23}\right\rangle /\left\langle\sigma_{22}\right\rangle$ is on the same order as that from the typical cross section study in the beginning of this section. Fig. 3 clearly shows that the cutoff effect in the high $\mu^{2}$ and low $s$ region for the radiative process relative to the elastic process (Fig. 2). The ratio of the rates per unit volume, $w_{23} / w_{22}$, is even smaller than $\left\langle\sigma_{23}\right\rangle /\left\langle\sigma_{22}\right\rangle$. It returns a value around $12 \%$ and shows the effect of particle distribution on the rates. There certainly can be alternative models for radiative processes. A recent study by the Frankfurt group appears to agree qualitatively with our study [16]. If the radiative cross sections are smaller than previously expected from the Gunion-Bertsch formula, chemical equilibration will also be slower. However, as particle isotropization, kinetic equilibration, specific shear viscosity depend also on the momentum transfer, detailed studies are necessary to find out how they behave.

\section{Acknowledgements}

We thank J. Mayfield for helpful discussions. This research was supported by the U.S. National Science Foundation under Grant No. PHY-0970104.

\section{References}

[1] U. W. Heinz and R. Snellings, arXiv:1301.2826 [nucl-th].

[2] Z. Xu and C. Greiner, Phys. Rev. C 71, 064901 (2005) [hep-ph/0406278].

[3] M. Asakawa, S. A. Bass and B. Müller, Phys. Rev. Lett. 96, 252301 (2006) [hep-ph/0603092].

[4] B. Zhang, L. -W. Chen and C. M. Ko, J. Phys. G 35, 065103 (2008) [arXiv:0705.3968 [nucl-th]].

[5] P. Huovinen and D. Molnar, Phys. Rev. C 79, 014906 (2009) [arXiv:0808.0953 [nucl-th]].

[6] A. El, Z. Xu and C. Greiner, Phys. Rev. C 81, 041901 (2010) [arXiv:0907.4500 [hep-ph]].

[7] G. S. Denicol, T. Koide and D. H. Rischke, Phys. Rev. Lett. 105, 162501 (2010) [arXiv:1004.5013 [nucl-th]].

[8] M. Martinez and M. Strickland, Nucl. Phys. A 848, 183 (2010) [arXiv:1007.0889 [nucl-th]].

[9] K. Dusling, T. Epelbaum, F. Gelis and R. Venugopalan, Phys. Rev. D 86, 085040 (2012) [arXiv:1206.3336 [hep-ph]].

[10] F. Gelis and T. Epelbaum, arXiv:1307.2214 [hep-ph].

[11] Z. Xu and C. Greiner, Phys. Rev. Lett. 100, 172301 (2008) [arXiv:0710.5719 [nucl-th]].

[12] J. -W. Chen, H. Dong, K. Ohnishi and Q. Wang, Phys. Lett. B 685, 277 (2010) [arXiv:0907.2486 [nucl-th]].

[13] B. Zhang and W. A. Wortman, Phys. Lett. B 693, 24 (2010) [arXiv:1006.0270 [nucl-th]].

[14] J. -W. Chen, J. Deng, H. Dong and Q. Wang, Phys. Rev. D 83, 034031 (2011) [Erratum-ibid. D 84, 039902 (2011)] [arXiv:1011.4123 [hep-ph]].

[15] B. Zhang, J. Phys. Conf. Ser. 420, 012035 (2013) [arXiv:1208.1224 [nucl-th]].

[16] O. Fochler, J. Uphoff, Z. Xu and C. Greiner, arXiv:1302.5250 [hep-ph].

[17] X. -G. Huang and J. Liao, arXiv:1303.7214 [nucl-th].

[18] J. F. Gunion and G. Bertsch, Phys. Rev. D 25, 746 (1982).

[19] X. -N. Wang, M. Gyulassy and M. Plümer, Phys. Rev. D 51, 3436 (1995) [hep-ph/9408344].

[20] T. Gottschalk and D. W. Sivers, Phys. Rev. D 21, 102 (1980).

[21] F. A. Berends, R. Kleiss, P. De Causmaecker, R. Gastmans and T. T. Wu, Phys. Lett. B 103, 124 (1981). 